\title{
SEPARATION AXIOMS INQUAD TOPOLOGICAL SPACES
}

\author{
U.D. Tapi, Ranu Sharma, Bhagyashri A. Deole \\ Department of Applied Mathematics \\ and Computational Science, SGSITS, Indore (M.P.) \\ Department of Applied Mathematics \\ and Computational Science, SGSITS, Indore (M.P.) \\ Affiliated to D.A.V.V Indore (M.P), INDIA \\ Department of Applied Mathematics \\ and Computational Science, SGSITS, Indore (M.P.)
}

\begin{abstract}
Keywords: Quad topological spaces, space, $q-T_{0}$ space, $q-T_{1}$ space, $q-T_{2}$ space, $q-T_{3}$ regular space.
\end{abstract}

ABSTRACT. In this paper, we introduce separation axioms in quad topological space (q topological spaces) and study some of their properties.

\section{INTRODUCTION}

J .C. Kelly [2] introduced bitopological spaces in 1963. The study of tri-topological spaces was first initiated by Martin M. Kovar [3] in 2000,where a non empty set X with three topologies is called tri-topolgical spaces. N.F. Hameed \& Mohammed Yahya Abid [1] studied separation axioms in tritopological spaces. D.V. Mukundan [4] introduced the concept on topological structures with four topologies, quad topology ( 4 - tuple topology ) and defined new types of open (closed )sets. In this paper, we use q-open and q-closed sets defined by D.V. Mukundan [4 ] to explain the concept of separation axioms in quad topological spaces.

\section{PRILIMINARIES}

Definition 2.1 [4] :Let $\mathrm{X}$ be a nonempty set and $\tau_{1}, \tau_{2}, \tau_{3}$ and $\tau_{4}$ are general topologies on $\mathrm{X}$. Then a subset $A$ of space $X$ is said to be quad-open(q-open) set if $A \subset \tau_{1} \cup \tau_{2} \cup \tau_{3} \cup \tau_{4}$ and its complement is said to be q-closed and set $\mathrm{X}$ with four topologies called $\left(\mathrm{X}, \tau_{1}, \tau_{2}, \tau_{3}, \tau_{4}\right)$. q-open sets satisfy all the axioms of topology.

Definition2.2 [4]: A subset of a q-topological space (X, $\left.\tau_{1}, \tau_{2}, \tau_{3}, \tau_{4}\right)$ is called q- Neighborhood of a point $x \in X$ if and only if there exist q-open sets such that $x \subset X \subset A$.

Note 2.3[4] : We will denote the q-interior (resp. q-closure) of any subset ,say of by q-intA (qclA), where q-intA is the union of all q-open sets contained in A, and q-clA is the intersection of all q-closed sets containing A.

\section{SEPARATION AXIOMS IN QUAD TOPOLOGICAL SPACES}

Definition 3.1: A quad (q) topological space $\mathrm{X}$ is said to be $q-T_{0}$ space iff to given any pair of distinct points $\mathrm{x}, \mathrm{y}$ in $\mathrm{X}$, there exists a q-open set containing one of the points but not the other .

$$
\begin{aligned}
& \text { Example 3.2: } \quad \text { Let } \quad X=\{a, b, c\}, \quad \tau_{1}=\{X, \emptyset,\{a\}\}, \tau_{2}=\{X, \emptyset,\{a\},\{a, b\}\}, \\
& \tau_{3}=\{X, \emptyset,\{a, b\}\}, \tau_{4}=\{X, \emptyset\} \text { all q-open sets are } X, \emptyset,\{a\},\{a, b\} \text { so }\left(\mathrm{X}, \tau_{1}, \tau_{2}, \tau_{3}, \tau_{4}\right) \text { is q- } \\
& \text { space. }
\end{aligned}
$$

Theorem 3.3: If $\{\mathrm{x}\}$ is q-open for some $x \in X$ then $x \notin q-c l\{y\}$, for all $y \neq x$. 
Proof: Let $\{\mathrm{x}\}$ be a q-open for some $x \in X$, then $X-\{x\}$ is q-closed and $x \notin X-\{x\}$. If $x \in q-c l\{y\}$, for some $y \neq x$., then $\mathrm{y}, \mathrm{x}$ both are in all the $\mathrm{q}$-closed sets containing $\mathrm{y}$, so $x \in X-\{x\}$ which is contradiction, hence $x \notin q-\operatorname{cl}\{y\}$.

Theorem 3.4: In any q-topological space $X$, any distinct points have distinct q-closures.

Proof: Let $x, y \in X$ with $x \neq y$, \& let $A=\{x\}^{c}$ hence $q-\operatorname{cl}(A)=A$ or $X$. Now if $\operatorname{cl}(A)=A$ then $\mathrm{A}$ is q-closed so $X-A=\{x\}$ is q-open \& not containing y. So by theorem(3.3) $x \notin q-\operatorname{cl}(\{y\}) \& y \in q-\operatorname{cl}(\{y\})$ which implies that $q-\operatorname{cl}(\{y\})$ and $q-\operatorname{cl}(\{x\})$ are distinct. If $q-\operatorname{cl}(A)=X$ then $\mathrm{A}$ is q-open, hence $\{\mathrm{x}\}$ is q-closed, which mean that $q-\operatorname{cl}(\{x\})=\{x\}$ which is not equal to $q-\operatorname{cl}(\{y\})$.

Theorem 3.5: In any quad topological space X, if distinct points have distinct $q-T_{0}$ closures then $\mathrm{X}$ is q- space .

Proof: Let $x, y \in X_{\text {with }} x \neq y$, with $q-\operatorname{cl}(\{y\})$ is not equal to $q-\operatorname{cl}(\{x\})$, hence there exists $z \in X$ such that $z \in q-\operatorname{cl}(\{x\})$, but $z \notin q-\operatorname{cl}(\{y\})$ or $z \in q-\operatorname{cl}(\{y\})$ but $z \notin q-\operatorname{cl}(\{x\})$. Now, without loss of generality, let $z \in q-\operatorname{cl}(\{x\})$, but $z \in q-\operatorname{cl}(\{y\})$. If $x \in q-\operatorname{cl}(\{y\})$, then $q-\operatorname{cl}(\{x\})$ is contained in $q-\operatorname{cl}(\{y\})$ hence $z \notin q-\operatorname{cl}(\{y\})$. which is a contradiction, this mean that $x \notin q-\operatorname{cl}(\{y\})$ hence $x \in q-\operatorname{cl}\left(\{y\}^{c}\right)$, hence $\mathrm{X}$ is $q-T_{0}$ space.

Definition 3.6: A quad -topological space $X$ is said to be $\mathrm{q}^{-} \mathrm{T}_{1}$ space iff to given any pair of distinct point $x \& y$ of $X$ there exist two q-open sets $U, V$ such that $x \in U, y \notin U \& y \in V, x \notin V$.

$$
\begin{aligned}
& \text { Example 3.7: } \quad \text { Let } \quad \mathrm{X}=\{\mathrm{a}, \mathrm{b}, \mathrm{c}\}, \tau_{1}=\{\mathrm{X}, \emptyset,\{\mathrm{a}\},\{\mathrm{a}, \mathrm{b}\}\}, \tau_{2}=\{\mathrm{X}, \emptyset,\{\mathrm{a}\}\}, \\
& \tau_{3}=\{\mathrm{X}, \emptyset,\{\mathrm{a}, \mathrm{b}\}\}, \tau_{4}=\{X, \emptyset,\{b\}\} \text { all q-open sets are } X, \emptyset,\{a\},\{b\},\{a, b\} \text { so }\left(\mathrm{X}, \tau_{1}, \tau_{2}, \tau_{3}, \tau_{4}\right) \\
& \text { is q-T } \mathrm{T}_{1} \text { space. }
\end{aligned}
$$

Theorem 3.8: Every q- $\mathrm{T}_{1}$ space is a $q-T_{0}$ space.

Proof : Follows from the definition of $\mathrm{q}^{-} \mathrm{T}_{1}$ space.

Following example shows that the converse of theorem (3.8) is not true.

Example 3.9: Let $\quad X=\{a, b, c\}, \quad \tau_{1}=\{X, \emptyset,\{a\},\{a, b\},\{a, c\}\}, \tau_{2}=\{X, \emptyset,\{a\}\}$, $\tau_{3}=\{X, \emptyset,\{a, c\}\}, \tau_{4}=\{X, \emptyset,\{a, b\}\}$, all q-open sets are $X, \emptyset,\{a\},\{a, b\},\{a, c\}$ so (X, $\left.\tau_{1}, \tau_{2}, \tau_{3}, \tau_{4}\right)$ is $q-T_{0}$ space but it is not $\mathrm{q}-\mathrm{T}_{1}$ space.

Definition 3.10: A quad topological space $X$ is said to be $q-T_{2}$ space if and only if for $x, y \in X, x \neq y$ there exist two disjoint q-open sets $\mathrm{U}, \mathrm{V}$ in $\mathrm{X}$ such that $x \in U \& y \in V$.

Example 3.11: Let $=\{a, b, c\}, \tau_{1}=\{X, \emptyset,\{a\},\{a, b\},\{a, c\}\}, \tau_{2}=\{X, \emptyset,\{b\},\{b, c\}\}$, $\tau_{3}=\{X, \emptyset,\{c\}\{a, c\}\}, \tau_{4}=\{X, \emptyset,\{a\}\}$ all q-open sets are $, X, \emptyset,\{a\},\{b\},\{c\},\{a, b\}\{a, c\},\{b, c\}$ so $\left(\mathrm{X}, \tau_{1}, \tau_{2}, \tau_{3}, \tau_{4}\right)$ is $\mathrm{q}-T_{2}$ space.

Theorem 3.12: Every ${ }^{\mathrm{q}-T_{2}}$ space is $\mathrm{q}-\mathrm{T}_{1}$ space .

Proof: Let $\mathrm{X}$ is $\mathrm{q}^{-T_{2}}$ space and let $\mathrm{x}$, $\mathrm{y}$ in $\mathrm{X}$ with $x \neq y$, so by hypothesis there exist two disjoint q-open ,say $\mathrm{U}, \mathrm{V}$ such that $x \in U \& y \in V$ but $U \cap V=\emptyset$ hence $x \notin \mathrm{V} \& \quad y \notin \mathrm{U}_{\mathrm{i} . \mathrm{e}} \mathrm{X}$ is q- $\mathrm{T}_{1}$ space. 
Definition 3.13: A quad topological space $X$ is said to be q-regular space if and only if for each qclosed set $\mathrm{F} \&$ each point $x \notin \mathrm{F}$, there exist disjoint q-open sets $\mathrm{U} \& \mathrm{~V}$ such that $x \in U \& F \subset V$. Example 3.14 : Let $X=\{a, b, c\}, \tau_{1}=\{X, \emptyset,\{a\}\}, \tau_{2}=\{X, \emptyset,\{b, c\}\}$ $\left.\tau_{3}=\{X, \emptyset,\{a\},\{b, c\}\}, \tau_{4}=\{X, \emptyset\}\right\}$, all q-open sets are $X, \emptyset,\{a\},\{b, c\}$ and q-closed sets are $\emptyset, X,\{b, c\},\{a\}$ so $\left(\mathrm{X}, \tau_{1}, \tau_{2}, \tau_{3}, \tau_{4}\right)$ is q-regular space.

Definition 3.15: A q-regular $T_{1}$ space is called a $\mathrm{q}^{-} T_{3}$ space

Theorem 3.16: Every $\mathrm{q}^{-} T_{3}$ space is a $\mathrm{q}^{-} T_{2}$ space.

Proof: Let $\left(\mathrm{X}, \tau_{1}, \tau_{2}, \tau_{3}, \tau_{4}\right)$ be a ${ }^{\mathrm{q}-T_{3}}$ space and let $\mathrm{x}$, $\mathrm{y}$ be two distinct points in $\mathrm{X}$. Now by definition, $X$ is also a $T_{1}$ space $\&$ so $\{x\}$ is a closed set. Also $\mathrm{y} \notin\{x\}$. Since $\left(X, \tau_{1}, \tau_{2}, \tau_{3}, \tau_{4}\right)$ is a q-regular space, there exist open sets $G \& H$ such that $\{x\} \subset G, y \in H \& G \cap H=\emptyset$. Also $\{x\} \subset G \Rightarrow x \in G$. Thus $\mathrm{x}$, y belong respectively to disjoint open sets G\& H. According (X, $\left.\tau_{1}, \tau_{2}, \tau_{3}, \tau_{4}\right)$ is a space.

\section{CONCLUSION}

In this paper the idea of separation axioms in quad topological space were introduced .

\section{REFERENCES}

[1] Hameed N.F. \& Mohammed Yahya Abid,Certain types of separation axioms in Tri-topological spaces, Iraqi journal of science, Vol 52, No.2,2011,PP212-217.

[2] Kelly J.C, Bitopological spaces, Proc.LondonMath.Soc., 3 PP. 17-89, 1963.

[3] Kovar M., On 3-Topological version of Thet-Reularity, Internat. J. Math, Sci,Vol.23, No.6,393398, 2000.

[4] Mukundan D.V., Introduction to Quad topological spaces, Int. Journal of Scientific \& Engg. Research, Vol.4, Issue 7, 2483-2485, July-2013 